The field equations can be written as

$$
\begin{gathered}
\psi_{\mu \nu}=\partial_{\mu} \psi_{\nu}-\partial_{\nu} \psi_{\mu}+\frac{1}{2}\left[\gamma_{\mu}(\gamma \partial) \psi_{\nu}-\gamma_{\nu}(\gamma \partial) \psi_{\mu}\right] \\
+\frac{1}{3} i \sigma_{\mu \nu} \partial_{\lambda} \psi^{\lambda} \\
\partial_{\mu} \psi^{\mu \nu}=\frac{1}{2} m{ }^{2} \psi^{\nu} .
\end{gathered}
$$

They can be derived from the Lagrange function

$$
\begin{aligned}
& L=\operatorname{const}\left(\bar{\psi}^{\mu \nu} \cdot \partial_{\mu} \psi_{\nu}+\bar{\psi}_{\nu} \cdot \partial_{\mu} \psi^{\mu \nu}\right. \\
&\left.+\frac{1}{2} \bar{\psi}^{\mu \nu} \cdot \psi_{\mu \nu}-\frac{1}{2} m{ }^{2} \bar{\psi}^{\mu} \cdot \psi_{\mu}\right) .
\end{aligned}
$$

It is easy to show that the field variable $\psi_{\mu}$ satisfies

$$
\begin{gathered}
\partial_{\lambda} \psi^{\lambda}=0, \\
\left(\partial^{2}-m^{2}\right) \psi_{\mu}=0,
\end{gathered}
$$

rather than the Dirac-type field equations. It is obvious that $\psi_{\mu}$ can be expressed as

where $\psi_{\mu}{ }^{(a)}$ satisfies

$$
\psi_{\mu}=\psi_{\mu}{ }^{(1)}+\gamma_{5} \psi_{\mu}{ }^{(2)} \text {, }
$$

$$
\begin{gathered}
(-i \gamma \partial+m) \psi_{\mu}^{(a)}=0 \quad(a=1,2), \\
\gamma_{\mu} \psi^{\mu(a)}=0 .
\end{gathered}
$$

This reveals again that our system is the superposition of two spin- $\frac{3}{2}$ fields of opposite parities. The fact that the energy is not positive definite follows exactly from the same arguments. Similar results can be obtained for other halfinteger spins. Finally, we would like to point out that an analogous modification of Tung's theory by imposing some subsidiary conditions can also be applied to systems of half-integer spins.

It is my pleasure to thank Dr. J. Mandula for reading the manuscript, and $\mathrm{Dr}$. W. K. Tung for a prepublication copy of his paper.

\footnotetext{
*Work supported in part by U. S. Air Force Office of Scientific Research under Contract No. A.F.49(638)1380 .

$\dagger$ John Parker Fellow.

${ }^{1}$ W. K. Tung, Phys. Rev. Letters 16, 763 (1966); thesis, Yale University, 1966 (unpublished). A summary of earlier works were listed in these two references. D. L. Pursey, Ann. Phys. (N.Y.) 32, 157 (1965).

${ }^{2}$ Throughout this paper we use the following notations: $g_{\mu \nu}=(-1,1,1,1) ;\left\{\gamma_{\mu}, \gamma_{\nu}\right\}=-2 g_{\mu \nu} ;$ all Greek indices $\mu$, $\nu, \circ \circ$, vary from 0 to 3 . Repeated indices are to be summed over.

${ }^{3}$ S. J. Chang, Phys. Rev. 148, 1259 (1966).

${ }^{4}$ M. Fierz and W. Pauli, Proc. Roy. Soc. (London) $\underline{\mathrm{A} 173}, 211$ (1939).
}

\title{
PHOTOPRODUCTION OF SINGLE POSITIVE PIONS BETWEEN 1.2 AND 3 GeV
}

\section{G. Buschhorn, J. Carroll, R. D. Eandi,* P. Heide, R. Hübner, W. Kern,} U. Kötz, P. Schmüser, and H. J. Skronn Deutsches Elektronen Synchrotron DESY, Hamburg, Germany, and II. Institut für Experimentalphysik der Universität Hamburg, Hamburg, Germany (Received 6 September 1966)

\begin{abstract}
Differential cross sections for the reaction $\gamma+p \rightarrow n+\pi^{+}$are presented for incident photon energies between 1.2 and $3 \mathrm{GeV}$ and pion center-of-mass production angles of 15 to $50 \mathrm{deg}$.
\end{abstract}

We have measured the production of positive pions from the reaction $\gamma+p \rightarrow n+\pi^{+}$using a high-resolution magnetic spectrometer equipped with scintillation counter hodoscopes (Fig. 1). The pions were produced in a liquid-hydrogen target by a bremsstrahlung beam obtained from an internal target of the Deutsches Elektronen Synchrotron (DESY). The bremsstrahlung beam was defined by two collimators, each followed by a sweeping magnet. A gas-filled quantameter was used to measure the photon flux. ${ }^{1}$
The spectrometer magnets were mounted on a movable platform pivoted at the target center. Synchrotron magnets $(C 1$ and $C 2)$ bent the particles away from the photon beam and provided an angular focus in the horizontal plane at $H 1$. By means of the quadrupole doublet $(Q 1$ and $Q 2)$ and the bending magnets ( $M 1$ and M2) a momentum-dispersed image of $H 1$ was formed at $H 2$. Counter hodoscope $H 1$ measured the production angle of the particles with an effective resolution of $\pm 2.5 \mathrm{mrad}$. Hodoscope 


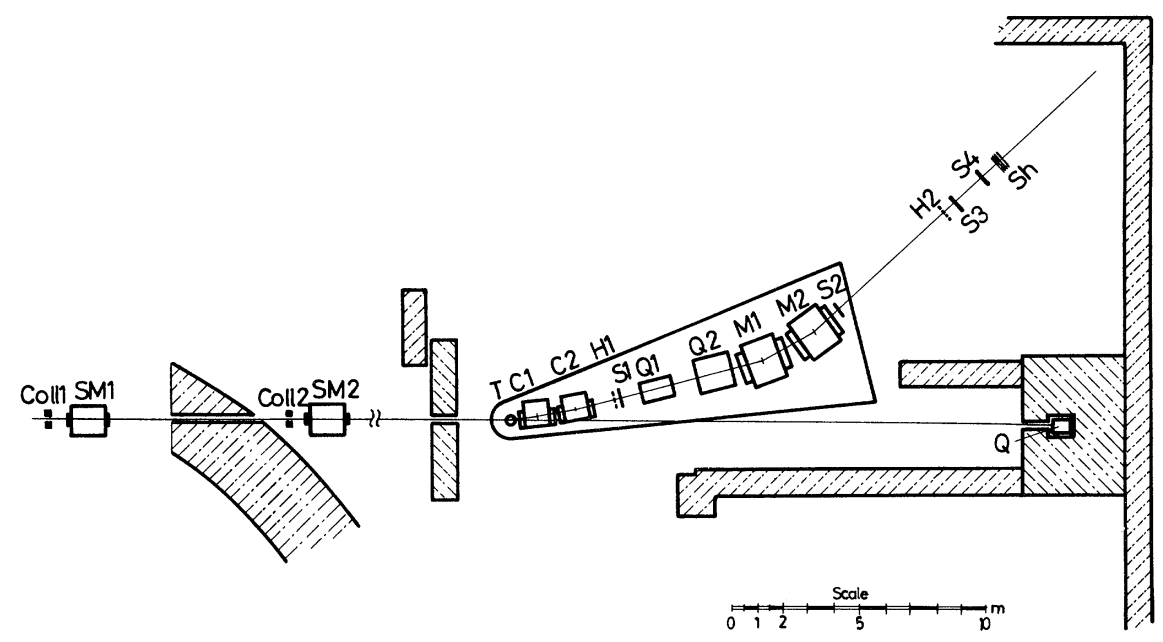

FIG. 1. Plan view of experimental arrangement.

H2 measured a linear combination of the particle's production angle $(\theta)$ and momentum $(p)$. By approximately matching the acceptance window of each $\mathrm{H} 2$ counter to the slope $(\partial p / \partial \theta)$ of the pion-photoproduction kinematics, we obtained a photon energy resolution of $\pm 0.4 \%$, which permitted the separation of single - from multiple-pion production processes up to our highest photon energy. To first order the resolution of this "sloped-window-type spectrometer"' is independent of target size.

The passage of a particle through the spectrometer was indicated by a coincidence $S_{1} S_{2} S_{3} S_{4}$ between the trigger counters. A measurement of the time of flight of the particle between $S_{1}$ and $S_{3}$ with a resolution of $2 \mathrm{nsec}$ (full width at half-maximum) permitted the separation of pions from protons up to our maximum pion momentum of $2.75 \mathrm{GeV} / c$. A lead-scintillator-sandwich shower counter was used to measure the positron contamination of the pion sample. This contamination was found to be negligible in all our measurements.

At each occurrence of the coincidence $\left(S_{1} S_{2} S_{3} S_{4}\right)$, the trajectory information from the hodoscopes, the particle time of flight, and the shower-counter status were recorded in the memory of a PDP-5 computer. The PDP- 5 served as a buffer during transfer of the data to magnetic tape and also provided a continous display of the data. Cross-section calculations and other data analysis were done on an IBM-7044 to which the PDP-5 was attached by a datalink.

The acceptance, $A=\int d \Omega(d p / p)$, for each pair- combination of hodoscope elements was calculated by a Monte Carlo technique which included the effects of (1) the finite size of the hydrogen target and photon beam, (2) multiple Coulomb scattering, (3) the stops determined by magnet apertures and counter sizes, and (4) pion decay in flight. The total acceptance of the spectrometer was $0.55 \times 10^{-4} \mathrm{sr}$. The error for the acceptances is estimated to be less than $5 \%$.

Cross sections have been measured at pion laboratory angles from 8 to $20 \mathrm{deg}$ in 2-deg intervals, and at ten photon energies between 1.2 and $3 \mathrm{GeV}$. For each laboratory angle and photon energy, the central momentum of the spectrometer was adjusted so that the kinematic region accessible only to single-pion production was approximately centered in the momentum-angle acceptance of the spectrometer. In the subsequent analysis of the data, only those events falling into the kinematic region for single-pion production and well away from the edge of the bremsstrahlung spectrum were used to calculate the cross sections. The cross sections presented here have been corrected for empty-target background (7\%), pion decay in flight (4-16\%), nuclear absorption (8\%), ambiguous events (8\%), and effects of a thick target on the shape of the bremsstrahlung spectrum.

The differential cross sections, $d \sigma / d \Omega$ c.m.' are given in Table I as a function of incident photon energy $E_{\gamma}$ and center-of-mass pion production angle $\theta_{\text {c.m. }}$. (In Table I and Figs. 2 and 3 , the errors given are only those associ- 
Table I. Center-of-mass differential cross section for the reaction $\gamma+p \rightarrow n+\pi^{+}$as a function of pion center-ofmass production angle $\theta^{*}$ and incident laboratory photon energy $E_{\gamma}$. The cross sections are in units of microbarns per steradian.

\begin{tabular}{|c|c|c|c|c|c|c|c|c|}
\hline$\underbrace{\theta *}_{(\mathrm{GeV})}$ & $15^{\circ}$ & $20^{\circ}$ & $25^{\circ}$ & $30^{\circ}$ & $35^{\circ}$ & $40^{\circ}$ & $45^{\circ}$ & $50^{\circ}$ \\
\hline 1.20 & $3.64 \pm 0.11$ & $4.03 \pm 0.13$ & $4.37 \pm 0.11$ & $4.36 \pm 0.14$ & $4.41 \pm 0.11$ & $4.06 \pm 0.20$ & & \\
\hline 1.34 & $2.93 \pm 0.10$ & $3.58 \pm 0.11$ & $3.77 \pm 0.09$ & $3.75 \pm 0.12$ & $3.55 \pm 0.09$ & $3.37 \pm 0.13$ & & \\
\hline 1.48 & $3.20 \pm 0.12$ & $3.13 \pm 0.09$ & $3.32 \pm 0.17$ & $2.87 \pm 0.08$ & $2.70 \pm 0.07$ & $2.44 \pm 0.07$ & & \\
\hline 1.62 & $3.40 \pm 0.14$ & $2.71 \pm 0.09$ & $2.87 \pm 0.14$ & $2.43 \pm 0.07$ & $2.03 \pm 0.06$ & $1.61 \pm 0.04$ & $1.05 \pm 0.11$ & \\
\hline 1.77 & $3.28 \pm 0.14$ & $2.90 \pm 0.08$ & $2.72 \pm 0.07$ & $2.36 \pm 0.06$ & $1.78 \pm 0.06$ & $1.39 \pm 0.04$ & $1.17 \pm 0.06$ & \\
\hline 1.98 & $2.98 \pm 0.15$ & $2.87 \pm 0.07$ & $2.47 \pm 0.06$ & $2.04 \pm 0.05$ & $1.64 \pm 0.06$ & $1.19 \pm 0.03$ & $1.00 \pm 0.03$ & \\
\hline 2.18 & $2.60 \pm 0.17$ & $2.44 \pm 0.06$ & $2.35 \pm 0.06$ & $1.86 \pm 0.07$ & $1.42 \pm 0.05$ & $1.04 \pm 0.03$ & $0.69 \pm 0.02$ & $0.38 \pm 0.07$ \\
\hline 2.38 & $2.13 \pm 0.23$ & $1.91 \pm 0.06$ & $1.71 \pm 0.05$ & $1.51 \pm 0.05$ & $1.04 \pm 0.03$ & $0.81 \pm 0.03$ & $0.58 \pm 0.02$ & $0.41 \pm 0.03$ \\
\hline 2.63 & $2.14 \pm 0.09$ & $1.64 \pm 0.06$ & $1.38 \pm 0.04$ & $1.19 \pm 0.04$ & $0.83 \pm 0.03$ & $0.67 \pm 0.03$ & $0.42 \pm 0.02$ & $0.33 \pm 0.02$ \\
\hline 2.88 & & $1.46 \pm 0.06$ & $1.14 \pm 0.05$ & $1.07 \pm 0.08$ & $0.80 \pm 0.04$ & $0.53 \pm 0.03$ & $0.37 \pm 0.02$ & $0.26 \pm 0.01$ \\
\hline 3.16 & & & & & & & $0.25 \pm 0.03$ & $0.22 \pm 0.03$ \\
\hline 3.29 & & & & & & & $0.25 \pm 0.02$ & $0.17 \pm 0.01$ \\
\hline
\end{tabular}

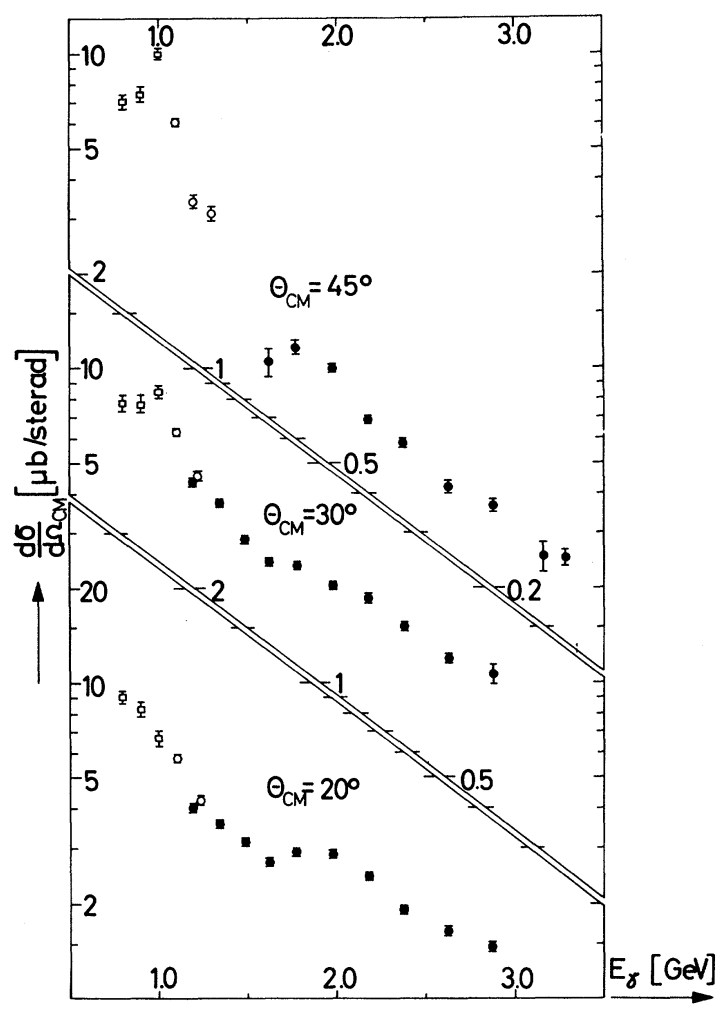

FIG. 2. Center-of-mass differential cross sections for $\gamma+p \rightarrow n+\pi^{+}$as a function of incident laboratory photon energy, $E_{\gamma}$. $\bullet$, this experiment; $\bigcirc$, J. R. Kilner, thesis, California Institute of Technology, 1963 (unpublished); $\square$, F. P. Dixon, thesis, California Institute of Technology, 1960 (unpublished).

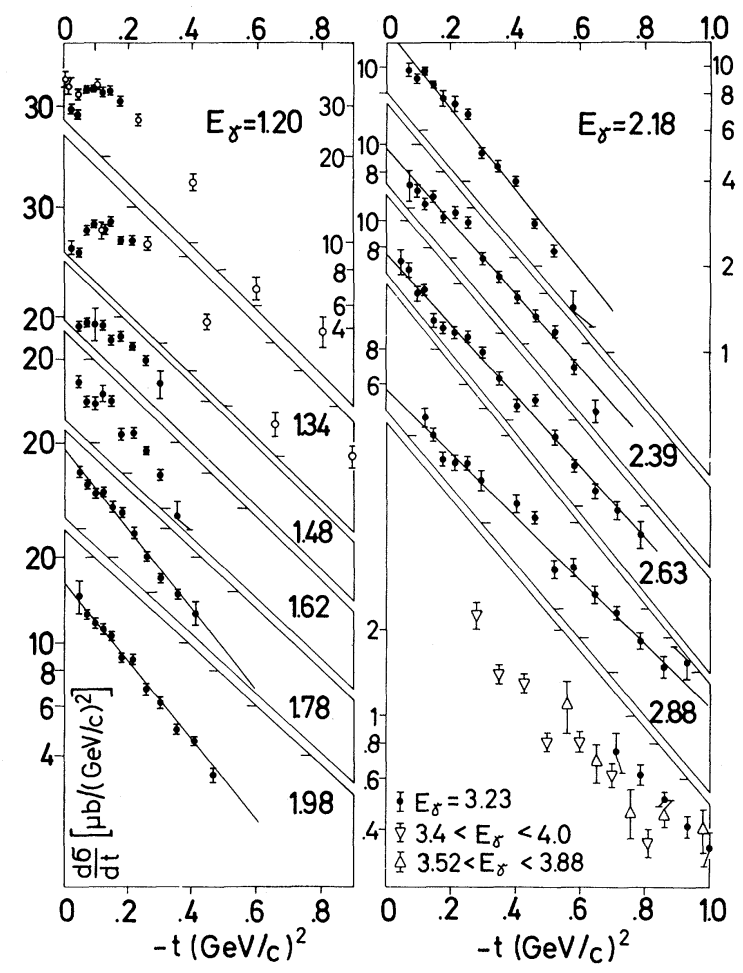

FIG. 3. $d \sigma / d t$ as a function of $-t$ ( $E_{\gamma}$ given in $\left.\mathrm{GeV}\right)$. - this experiment; $\bigcirc$, J. R. Kilner, thesis, California Institute of Technology, 1963 (unpublished), $E_{\gamma}$ $=1.20$ and $1.30 \mathrm{GeV} ; \nabla$ and $\Delta, \mathrm{V}$. B. Elings, K. J. Cohen, D. A. Garelick, S. Homma, R. A. Lewis, P.D. Luckey, and L. S. Osborne, Phys. Rev. Letters 16, 474 (1966). The straight lines drawn through the data points are described in the text (see also Table II). 
ated with counting statistics; the over-all systematic errors in the cross sections are estimated to be less than $9 \%$.) These cross sections have been averaged over angular intervals of $\Delta \theta_{\text {c.m. }}=5^{\circ}$ and energy intervals ranging from $\Delta \dot{E}_{\gamma}=0.1 \mathrm{GeV}$ at $E_{\gamma}=1.2 \mathrm{GeV}$ to $\Delta E_{\gamma}$ $=0.15 \mathrm{GeV}$ at $E_{\gamma}=3.0 \mathrm{GeV}$. We have determined that this choice of intervals does not appreciably change the energy-angle structure of the cross sections. The energy behavior of the cross sections at $\theta_{\text {c.m. }}=20,30$, and $45^{\circ}$ is shown in Fig. 2 with some California Institute of Technology data of Kilner ${ }^{3}$ and Dixon ${ }^{4}$. The dominant feature of these cross sections is a rapid decrease with energy. Between 1.5and 2.0-GeV incident photon energy we observe some structure in $d \sigma / d \Omega$ c.m. which might be due to the nucleon isobars of masses 1924 and $2190 \mathrm{MeV}$. The angular distributions show a fairly uniform decrease with angle at all energies above $1.7 \mathrm{GeV}$. However, at the smallest energies, 1.2 and $1.34 \mathrm{GeV}$, the angular distributions begin to turn over below $\simeq 30^{\circ}$, in agreement with Kilner. ${ }^{3}$ The intermediate energies of 1.48 and $1.62 \mathrm{GeV}$ apparently represent a transition region between these two types of behavior.

In Fig. 3 the data are presented in the form of $d \sigma / d t$ as a function of $-t$ at constant $E_{\gamma}$. These data have been averaged over energy intervals as given above and over intervals in $-t$ ranging from $\Delta t=0.02$ to $0.075(\mathrm{GeV} / c)^{2}$ at $-t=0.02$ and $1.0(\mathrm{GeV} / c)^{2}$, respectively. At photon energies above $1.75 \mathrm{GeV}$ we have fitted the data with the expression $d \sigma / d t=A$ $\times \exp (B t)$. The results of this fitting procedure are given in Table II. Although this parametrization represents the dominant behavior of

Table II. Results of least-squares fitting our cross sections with the form $d \sigma / d t=A \exp (B t)$. The total chi squared, $\chi^{2}$, and the number of degrees of freedom, $D$, are given for each fit.

\begin{tabular}{cccrr}
\hline $\begin{array}{c}E_{\gamma} \\
(\mathrm{GeV})\end{array}$ & $\begin{array}{c}A \\
{\left[\mu \mathrm{b} /(\mathrm{GeV} / c)^{2}\right]}\end{array}$ & $\begin{array}{c}B \\
{\left[(\mathrm{GeV} / c)^{-2}\right]}\end{array}$ & \multicolumn{1}{c}{$\chi^{2}$} & \multicolumn{1}{c}{$D$} \\
\hline 1.78 & $19.10 \pm 0.45$ & $3.32 \pm 0.11$ & 17.8 & 9 \\
1.98 & $16.20 \pm 0.40$ & $3.22 \pm 0.10$ & 9.7 & 9 \\
2.18 & $13.70 \pm 0.31$ & $3.31 \pm 0.08$ & 47.1 & 11 \\
2.39 & $9.65 \pm 0.25$ & $2.94 \pm 0.08$ & 32.0 & 12 \\
2.63 & $7.56 \pm 0.19$ & $2.86 \pm 0.07$ & 27.7 & 15 \\
2.88 & $5.78 \pm 0.20$ & $2.55 \pm 0.07$ & 20.6 & 14 \\
\hline
\end{tabular}

the cross sections rather well, the fits obtained at some energies are poor. The deviations of the experimental points from the exponential form show no apparent systematic behavior and may be due to small systematic errors in the data. The values of $B$ given in Table II indicate that the exponential fall-off of the cross section becomes less rapid as the incident photon energy increases. Elings et al. ${ }^{5}$ obtained $B=3.0 \pm 0.3(\mathrm{GeV} / c)^{-2}$ from their $\pi^{+}$photoproduction data which is averaged over photon energies from 3.52 to $3.88 \mathrm{GeV}$. In elastic pion-nucleon scattering, however, larger values of $B\left[\simeq 8(\mathrm{GeV} / c)^{-2}\right]$ are observed at comparable momentum transfers. ${ }^{6}$ We also note that the values of $A$ given in Table II show an approximately exponential decrease with increasing photon energy.

We wish to acknowledge the continued interest and help received from Professor W. Jentschke and Professor P. Stähelin. The contribution of Dr. W. Bertram during the early stages of the experiment is gratefully acknowledged. Sincere thanks go to Mr. F. Akolk for setting up the PDP system and interfaces, and to Mr. P. E. Kuhlmann for writing the necessary IBM system programs. Finally, we thank Dr. H. O. Wüster and the DESY computation center staff for their efforts, and Mr. H. Kumpfert and the synchrotron operating crew for their efficient running of the machine.

*North Atlantic Treaty Organization Postdoctoral Fellow, on leave from University of California Lawrence Radiation Laboratory.

${ }^{1}$ R. R. Wilson, Nucl. Instr. 1, 101 (1957). The calibration constant for the quantameter used is $(3.49 \pm 0.06)$ $\times 10^{18} \mathrm{MeV} / \mathrm{C}$ as measured by A. Ladage and H. Pingel; see Deutsches Electronen Synchrotron Report No. 65/12, 1965 (unpublished).

${ }^{2}$ Klaus G. Steffen, High Energy Beam Optics (Interscience Publishers, Inc., New York, 1965), p. 133.

${ }^{3} \mathrm{~J}$. R. Kilner, thesis, California Institute of Technology, 1963 (unpublished).

${ }^{4}$ F. P. Dixon, thesis, California Institute of Technology, 1960 (unpublished).

${ }^{5}$ V. B. Elings, K. J. Cohen, D. A. Garelick, S. Homma, R. A. Lewis, P. D. Luckey, and L. S. Osborne, Phys. Rev. Letters 16, 474 (1966).

${ }^{6}$ C. C. Ting, L. W. Jones, and M. L. Perl, Phys. Rev. Letters 9 , 468 (1962); D. E. Damouth, L. W. Jones, and M. L. Perl, ibid. 11, 287 (1963); C. T. Coffin, N. Dikmen, L. Ettlinger, D. Meyer, A. Saulys, K. Terwilliger, and D. Williams, ibid. 15, 838 (1965). 